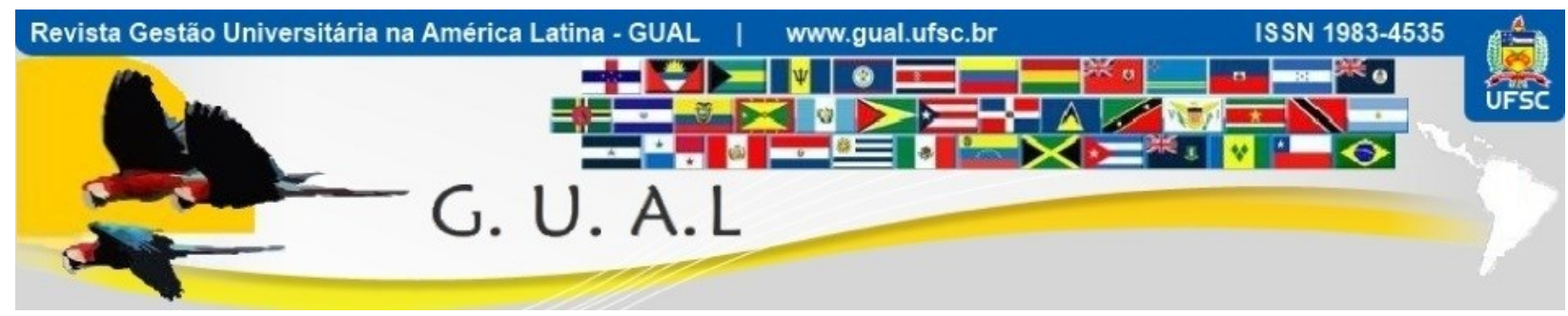

DOI: http://dx.doi.org/10.5007/1983-4535.2018v11n2p319

\title{
A UTILIZAÇÃO DAS PRÁTICAS DE GESTÃO DO CONHECIMENTO E FACILITADORES ESTRATÉGICOS EM UMA INSTITUIÇÃO DE ENSINO SUPERIOR
}

\section{THE USE OF KNOWLEDGE MANAGEMENT PRACTICES AND STRATEGIC FACILITATORS IN AN INSTITUTION OF HIGHER EDUCATION}

Luiz Carlos da Silva Oliveira, Doutorando

Universidade de Brasília - UnB Universidade Federal de Goiás - UFG lucaoliveira@uol.com.br

Eda Castro Lucas de Souza, Doutora

Universidade de Brasilia - UnB edaluca@gmail.com

Recebido em 17/novembro/2016

Aprovado em 22/fevereiro/2018

Sistema de Avaliação: Double Blind Review

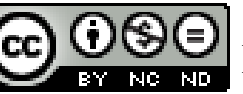

Esta obra está sob uma Licença Creative Commons Atribuição-Uso. 


\title{
RESUMO
}

Este estudo visa verificar a utilização de facilitadores estratégicos como instrumento de apoio às práticas de gestão do conhecimento em instituição de ensino superior. É apresentado um referencial teórico sobre prática de gestão do conhecimento e os facilitadores estratégicos: liderança, cultura, tecnologia de informação e desempenho. A natureza da pesquisa é quantitativa e o instrumento utilizado foi uma versão modificada da ferramenta de avaliação da gestão do conhecimento desenvolvido pela Arthur Andersen Consulting e o Centro de Qualidade e Produtividade Americana (APQC) e os dados foram coletados entre os meses de setembro a novembro de 2015. Os resultados indicam que existem diferenças significativas no grau de importância e uso de práticas de gestão do conhecimento. Da mesma forma, a importância e a utilização dos principais facilitadores estratégicos revelam diferenças significativas, sugerindo que desempenho, cultura, liderança e tecnologia da informação são importantes para as práticas de gestão do conhecimento e os processos de conhecimento (geração, codificação e transferência) necessitam da tecnologia da informação como suporte de gestão e medição de desempenho.

Palavras-chave: Prática de Gestão do Conhecimento. Facilitadores Estratégicos. Instituição de Ensino Superior.

\begin{abstract}
This study aims to verify the use of strategic facilitators as a tool to support knowledge management practices in a higher education institution. It presents a theoretical reference on knowledge management practice and strategic facilitators: leadership, culture, information technology and performance. The nature of the research is quantitative and the instrument used was a modified version of the knowledge management evaluation tool developed by Arthur Andersen Consulting and the American Quality and Productivity Center (APQC) and the data were collected between September and November of 2015. The results indicate that there are significant differences in the degree of importance and use of knowledge management practices. Similarly, the importance and use of key strategic enablers reveal significant differences, suggesting that performance, culture, leadership, and information technology are important for knowledge management practices, and knowledge processes (generation, coding, and transfer) require technology of information such as management support and performance measurement.
\end{abstract}

Keywords: Knowledge Management Practice. Strategic Facilitators. Institution of Higher Education. 


\section{A UTILIZAÇÃO DAS PRÁTICAS DE GESTÃO DO CONHECIMENTO E FACILITADORES \\ ESTRATÉGICOS EM UMA INSTITUIÇÃO DE ENSINO SUPERIOR \\ DOI: http://dx.doi.org/10.5007/1983-4535.2018v11n2p319}

\section{INTRODUÇÃO}

As organizações necessitam desenvolver-se para oferecerem aos clientes, produtos e serviços que os satisfaçam, mantendo-se sempre atentos às novas exigências do mercado. Compreender tais mecanismos é crucial para atingir os objetivos estratégicos das organizações, bem como a excelência organizacional. A gestão do conhecimento envolve elaboração de estratégias organizacionais, no que diz respeito à estrutura, processos e sistemas, visando obter conhecimento necessário para a consecução dos objetivos da organização (CHOO, 2003).

Quando o foco são as instituições de ensino superior (IES), a necessidade de informação está diretamente relacionada aos seus processos acadêmicos. A execução alinhada de tais processos pode subsidiar a gestão de conhecimento da instituição e favorecer a construção de conhecimento. Nota-se que há crescente reconhecimento de que uma abordagem ampla e institucional para a gestão do conhecimento possibilitará às instituições de ensino superior (IES) tornarem mais eficazes em ambiente educativo dinâmico, com melhorias consideráveis em atividades de intercâmbio de conhecimentos e, posteriormente, o desempenho organizacional (SHARIMLLAH DEVI et al., 2007).

Ressalta-se que no contexto das instituições de ensino superior, os obstáculos e facilitadores para a implantação da gestão do conhecimento foram pesquisados anteriormentes por Eiriz, Simões e Gonçalves (2007). De acordo com Cuffa, Rojo e Mello (2014) os obstáculos e facilitadores envolvem também as questões do compartilhamento e da cultura das instituições e, tanto nas organizações quanto nas instituições a implantação dessa gestão requer metodologia, acuidade e responsabilidade por parte de todos os envolvidos no processo. Percebe-se também que estudos sobre facilitadores estratégicos de apoio à gestão do conhecimento tem recebido atenção considerável na literatura corporativa que enfocam estratégia, liderança, cultura organizacional, tecnologia da informação (TI), e medição de desempenho (CHONG, 2006; CHONG E LIN, 2009).

$\mathrm{Na}$ educação superior, a gestão do conhecimento ainda é conceito que carece de esclarecimento para que as práticas emergentes possam se diluir nesses modelos institucionais. Porém, aquelas já alinhadas às práticas de gestão do conhecimento percebem resultados importantes dessa utilização, dentre eles a melhoria significativa em processos, o desenvolvimento de uma cultura voltada ao conhecimento e a constituição de uma dinâmica 


\section{A UTILIZAÇÃO DAS PRÁTICAS DE GESTÃO DO CONHECIMENTO E FACILITADORES \\ ESTRATÉGICOS EM UMA INSTITUIÇÃO DE ENSINO SUPERIOR \\ DOI: http://dx.doi.org/10.5007/1983-4535.2018v11n2p319}

de inovação que permite um posicionamento competitivo no segmento de atuação (BUCHELE et al, 2016).

Estudo recente de Mousavizadeh et al. (2015) buscou integrar facilitadores importantes, tais como cultura e apoio à gestão para a avaliação dos benefícios derivados da prática de gestão do conhecimento. $\mathrm{O}$ interesse chave dentro desta discussão é como as práticas de gestão do conhecimento estão sendo utilizada nas organizações (MARIN, CORDIER, HAMEED, 2016). Assim, a seguinte questão é objeto de estudo: Como são utilizados os facilitadores estratégicos que apoiam às práticas de gestão do conhecimento em instituição de ensino superior?

Portanto, esta pesquisa visa alcançar os seguintes objetivos: (1) examinar o grau de importância de práticas de gestão do conhecimento e facilitadores estratégicos em instituições de ensino superior; (2) Verificar a existência de dicotomias entre a importância e a utilização das práticas de gestão do conhecimento e facilitadores estratégicos em instituições de ensino superior; e (3) identificar as relações entre as práticas de gestão do conhecimento e os facilitadores estratégicos em termos de importância e uso em instituições de ensino superior.

Este estudo está estruturado em cinco diferentes seções. A introdução contextualiza e estabelece os objetivos e estrutura do trabalho. A segunda apresenta uma revisão de literatura sobre as práticas de gestão do conhecimento nas IES e os facilitadores estratégicos a serem utilizados. A terceira enfoca o método da pesquisa, bem como a amostra e o instrumento utilizado; a quarta secção enfoca os resultados alcançados e a quinta as discussões e, por último, as conclusões da pesquisa.

\section{REVISÃO DE LITERATURA}

\subsection{PRÁTICAS DE GESTÃO DO CONHECIMENTO NAS IES}

Tendo em vista a importância do conhecimento, não é de surpreender que as organizações estejam dando destaque à descoberta de conhecimento e as formas de organizar, compartilhar, e aplicá-lo eficazmente visando tornarem-se competitivas e produtivas. Têm-se argumentado que o sucesso das atividades de gerenciamento de conhecimento em sua maior parte depende de quão eficiente e eficazmente o conhecimento tem sido utilizado (CHONG et al., 2000). A execução de práticas organizacionais da gestão de conhecimento está no cerne da criação de uma empresa baseada no conhecimento bem sucedido. 


\section{A UTILIZAÇÃO DAS PRÁTICAS DE GESTÃO DO CONHECIMENTO E FACILITADORES \\ ESTRATÉGICOS EM UMA INSTITUIÇÃO DE ENSINO SUPERIOR \\ DOI: http://dx.doi.org/10.5007/1983-4535.2018v11n2p319}

Hartung e Oliveira (2013) afirmam que a gestão de conhecimento representa uma forma das organizações identificarem os conhecimentos necessários para que seja possível aumentar a produtividade e a eficiência a longo prazo. Para os autores a organização adquire, compartilha e utiliza o conhecimento visando a sustentação e o alcance dos objetivos organizacionais. Assim a gestão do conhecimento é amplamente reconhecida como uma prática que envolve a geração, a codificação, e a transferência de conhecimento (CHONG e LIN, 2009).

A geração de conhecimento refere-se, basicamente, às subpráticas de busca, captura, e criação do conhecimento. Essa geração se dá através da descoberta, ou seja, os colaboradores desenvolvem novas maneiras de fazer as coisas (COUKOS-SEMMEL, 2002). A codificação engloba uma prática onde o conhecimento é codificado e armazenado em um formato razoável para que outras pessoas na organização possam acessá-la. O compartilhamento do conhecimento, segundo Davenport e Prusak, (2003) corresponde à transferência deste entre os membros da organização, seu entendimento e utilização pelos membros, valendo-se de duas estratégias: transferência espontânea (informal) ou estruturada (formal).

As instituições de educação superior têm sido tradicionalmente utilizadas como mecanismos de transferência para fornecer aos estudantes uma base de conhecimento que permitirá o seu desenvolvimento (TIPPINS, 2003). O processo de transferência do conhecimento ocorre pela interação entre alunos e docentes e, para isto, é necessário que o docente possua não só habilidades técnicas, mas também, um método de ensino capaz de estabelecer conexões entre teoria e prática.

Portanto, as funções do ensino superior, por natureza, têm sido consideradas como de conhecimento intensivo tendo como base a produção, codificação e distribuição (KENSKI, 2007). Nesse contexto sua gestão é definida como uma tentativa sistemática e deliberada de conceber e utilizar práticas do conhecimento em instituições de ensino superior e consiste em uma série de processos pelos quais o conhecimento dos acadêmicos é gerado, capturado, codificado, armazenado e compartilhado.

Schatzki (2005) afirma que as ações humanas são organizadas por meio de práticas. Assim, as múltiplas ações das pessoas se interligam (ou se organizam) e ganham uma identidade conjunta não de maneira aleatória, desordenada, mas em torno de uma dada prática por meio de uma estrutura de governança composta por três elementos fundamentais: entendimentos, regras, e estruturas teleoafetivas. 


\section{A UTILIZAÇÃO DAS PRÁTICAS DE GESTÃO DO CONHECIMENTO E FACILITADORES \\ ESTRATÉGICOS EM UMA INSTITUIÇÃO DE ENSINO SUPERIOR \\ DOI: http://dx.doi.org/10.5007/1983-4535.2018v11n2p319}

Os entendimentos estão relacionados ao "saber fazer", ou seja, à habilidade, à competência para "saber como" se pode (ou se deve) fazer as coisas, mas também o "saber como" identificar/entender as ações dos outros. As regras se referem às formulações explícitas, princípios, preceitos e instruções (leis, normas, regulamentos, procedimentos, critérios de decisão). A estrutura teleoafetiva, por sua vez, constitui um conjunto hierarquicamente organizado e normativo de fins (objetivos, interesses), meios para alcançálos (projetos, tarefas - ações) e, ainda, emoções (sentimentos, afetos, humor), que são aceitos e aconselhados, considerados como válidos ou legítimos na prática (SCHATZKI, 2005).

Portanto, o uso dessas práticas é apoiado por uma série de facilitadores como: estratégia, liderança, cultura organizacional, tecnologia da informação e desempenho. Tomados em conjunto, a gestão das práticas do conhecimento e as características dos principais facilitadores estratégicos são vitais para assegurar a implementação bem sucedida da gestão de conhecimento em instituições de ensino superior.

\subsection{FACILITADORES ESTRATÉGICOS}

A fim de assegurar o sucesso da execução da gestão de conhecimento, o uso de estratégias facilitadoras para apoiar as práticas de gestão do conhecimento é de suma importância. Na visão de Coukos-Semmel (2002) estes facilitadores formam um sistema que estimula os membros a desenvolverem conhecimentos e superarem vários constrangimentos na realização das diversas práticas de gestão do conhecimento. Os quatro principais facilitadores estratégicos serão discutidos nas subseções a seguir:

\subsubsection{Liderança}

Estudos de Mintzberg (1998) discute os efeitos da atuação do líder em equipes baseadas em conhecimento, considerando que este público responde à inspiração e não à supervisão. Já Taylor (2004) discute os processos de liderança necessários para a retenção de talentos em organizações, apontando que a retenção de talentos no contexto da liderança garante a competitividade e o desempenho organizacional.

Barbuto (2005) propôs que o processo de liderança ocorre de maneira transacional ou transformacional. A liderança transacional é baseada na autoridade burocrática e legitimidade dentro da organização. Líderes transacionais enfatizam padrões de trabalho, atribuições e tarefas orientadas para os objetivos. Eles também tendem a se concentrar na conclusão da 


\section{A UTILIZAÇÃO DAS PRÁTICAS DE GESTÃO DO CONHECIMENTO E FACILITADORES \\ ESTRATÉGICOS EM UMA INSTITUIÇÃO DE ENSINO SUPERIOR \\ DOI: http://dx.doi.org/10.5007/1983-4535.2018v11n2p319}

tarefa e na disciplina do funcionário, confiam em recompensas organizacionais e punições para influenciar o desempenho.

A liderança transformacional se caracteriza como um processo que motiva seguidores apelando aos ideais mais elevados e aos valores morais. Os líderes transformacionais devem ser capazes de definir e articular uma visão para suas organizações e os seguidores deve aceitar a credibilidade do líder (LOPES e FIALHO, 2014).

Kluge et al. (2001) apontam que embora os líderes em todos os níveis de uma organização tenham papéis excepcionais e urgentes a desempenhar. A gestão do conhecimento é predominantemente significativa para o executivo-chefe (CEO) envolver-se no processo de compartilhamento de conhecimento. Este envolvimento é vital, porque o CEO dá o tom e estabelece as políticas em uma organização.

Portanto, a liderança refere-se à capacidade dos líderes de uma organização de alinhar a gestão do conhecimento à estratégia organizacional, identificar oportunidades, promover o valor das práticas de gestão do conhecimento, comunicar melhores estratégias, facilitar a evolução da organização de aprendizagem, e fornecer métricas para avaliar o impacto do conhecimento. É por esta razão que pesquisadores afirmam que a liderança dos gestores e o compromisso são fatores crítico de sucesso na consecução de um projeto de gestão do conhecimento ( HOLSAPPLE e JOSHI, 2002).

\subsubsection{Cultura Organizacional}

O conceito de cultura tem sido utilizado em diversos contextos e com acentuadas diferenças de significado. Essa variação decorre da perspectiva utilizada e do que se assume como essencial: crenças; pressupostos básicos; valores compartilhados; ideologia; entendimentos significativos; programas coletivos da mente; e outros (TRICE e BEYER, 1984). Esse conceito pode ser concebido na complexidade e multidimensionalidade do conjunto, o qual constitui a vida em comum dos grupos sociais, ou seja, os fatores relacionados ao modo de pensar, agir e sentir compartilhados por grupos de pessoas em sua coletividade (PIRES e MACÊDO, 2006).

A importância da cultura para a gestão do conhecimento está relacionada com as práticas da organização, tendo em vista que a cultura organizacional possui quatro funções: fornecer aos membros da organização senso de identidade; aumentar o comprometimento organizacional; reforçar os valores organizacionais e, servir como mecanismo de controle 


\section{A UTILIZAÇÃO DAS PRÁTICAS DE GESTÃO DO CONHECIMENTO E FACILITADORES \\ ESTRATÉGICOS EM UMA INSTITUIÇÃO DE ENSINO SUPERIOR \\ DOI: http://dx.doi.org/10.5007/1983-4535.2018v11n2p319}

para moldar o comportamento dos atores da organização (NELSON e QUICK, 2011). Essa cultura é definida, também, como os padrões de valores e crenças que auxiliam as pessoas a entenderem o funcionamento organizacional e, assim, fornece-lhes normas de comportamento na organização.

Na visão de Heydari et al (2015) a motivação cultural, o sentimento de pertença à organização, a confiança, o respeito à necessidade de compartilhar, desenvolver e usar o conhecimento são necessários para o sucesso da gestão do conhecimento. Entretanto, o problema cultural é considerado um desafio na utilização das práticas de gestão em IES, porque, em vez de considerar o conhecimento como um ativo que aumenta de valor quando compartilhado, muitos membros do corpo docente consideram o conhecimento como propriedade, algo que não é compartilhado livremente. Assim, a cultura organizacional é um fator crítico que influencia o sucesso da implementação da gestão de conhecimento através do apoio às práticas eficazes nas organizações.

\subsubsection{Tecnologia da Informação}

Ao longo dos tempos, a tecnologia da informação passou a ser considerada um dos principais elementos dentro das organizações, passando a atuar em processos operacionais, táticos e estratégicos, como principal meio para obter desempenho e aumentar a efetividade das empresas.

A utilização da tecnologia da informação é uma das ferramentas que podem trazer um diferencial, e possibilitar vantagem competitiva. A grande variedade de informações e tecnologias convergentes interligadas, utilizadas para processamento de informações, está exigindo dos administradores maior conhecimento do valor estratégico da tecnologia da informação, suas peculiaridades e melhores práticas (ALBERTIN, 2001).

No contexto da educação superior a tecnologia da informação é uma realidade que tem provocado mudanças na construção de propostas pedagógicas que expressem um processo interativo e colaborativo visando a uma formação que permita reunir a utilização de tecnologias e conhecimento (BRENNAND, 2006).

Derntl e Motschning-Pitrik (2005) argumentam que as tecnologias de informação e comunicação possuem potencial para desempenhar um papel significativo com uma aproximação mais efetiva, em termos de maior aprofundamento dos processos de aprendizagem ao longo da vida. A tecnologia tem mostrado ser capaz de dar o apoio às 


\section{A UTILIZAÇÃO DAS PRÁTICAS DE GESTÃO DO CONHECIMENTO E FACILITADORES \\ ESTRATÉGICOS EM UMA INSTITUIÇÃO DE ENSINO SUPERIOR \\ DOI: http://dx.doi.org/10.5007/1983-4535.2018v11n2p319}

pessoas quanto à organização, transferência, e administração de informações, além de contribuir para promover um amplo espaço de estudo individual, interação em aula e experiências de aprendizagem. Para Kenski (2007) os vínculos entre conhecimento, poder e tecnologias estão presentes em todas as épocas e em todos os tipos de relações sociais. Logo, não há como deixar de lado os avanços tecnológicos, principalmente quando tratamos das relações de ensino e as práticas de gestão do conhecimento.

Diante desse quadro, a integração entre a gestão do conhecimento e tecnologia da informação aparece como um imperativo para atender a tais necessidades. Essa integração é complexa, pois envolve tanto o gerenciamento de ativos intangíveis de diferentes naturezas pessoas, conhecimentos tácitos, explícitos, individuais, organizacionais e de redes, quanto conhecimentos estruturais que servem de base tecnológica para a melhoria do fluxo dos bens intangíveis e de sistemas de informação com aplicativos que possibilitem o aumento da interação entre pessoas nos ambientes interno e externo.

Portanto, a visão da tecnologia de informação como infraestrutura para a gestão do conhecimento também é compartilhada por Carvalho (2003), para quem o principal papel da tecnologia da informação na gestão do conhecimento consiste em acelerar a velocidade de transferência do conhecimento. Apesar de ser uma importante ferramenta que possibilita a implantação da gestão do conhecimento nas organizações, a tecnologia da informação não deve ser confundida com a gestão do conhecimento propriamente dita, devendo ser considerada uma ferramenta de apoio à geração, ao armazenamento, ao controle e à difusão do conhecimento.

\subsubsection{Desempenho}

O desempenho das organizações tem recebido crescente atenção dos pesquisadores nas últimas décadas, mas não há consenso quanto sua operacionalização, pois definir, conceituar e medir o desempenho não tem sido uma tarefa fácil. De modo geral, os pesquisadores possuem percepções diferentes sobre desempenho organizacional. Neely et al (2000) salientam que o desempenho fornece ao gestor possibilidades de medir ações da organização, como sua capacidade produtiva, nível de eficiência e eficácia dos processos e os resultados das vendas. Antony e Bhattacharyya (2010) definem desempenho organizacional como medida que identifica como se dá a gestão das organizações, bem como o valor que entregam aos clientes e a outras partes interessadas. Já Huseein et al. (2014) referem-se ao desempenho 


\section{A UTILIZAÇÃO DAS PRÁTICAS DE GESTÃO DO CONHECIMENTO E FACILITADORES \\ ESTRATÉGICOS EM UMA INSTITUIÇÃO DE ENSINO SUPERIOR \\ DOI: http://dx.doi.org/10.5007/1983-4535.2018v11n2p319}

organizacional como resultado de vários processos organizacionais que ocorrem no curso das operações diárias da organização.

É importante destacar que Venkatraman e Ramanujan (1986) consideraram três aspectos de desempenho: o financeiro, o de negócios e o da efetividade organizacional. Esses autores sugeriram que os pesquisadores, além da utilização de indicadores financeiros, adotassem, também, indicadores operacionais ao medir o desempenho organizacional. Os indicadores operacionais incluem medidas como a introdução de novos produtos, qualidade do produto, eficácia de valor agregado e participação de mercado.

Para Maier e Remus (2002), o vínculo entre a gestão do conhecimento e o desempenho da organização não estão amplamente adotados na prática, devido à ausência de modelos que relacionem esforços de gestão do conhecimento, no sentido de processos orientados por estrutura e instrumentos organizacionais, atividades relativas à cultura empresarial, implementação de tecnologias e estratégia de negócios. Conforme esses autores, essa lacuna seria preenchida com a implementação de uma visão por processos, sendo a gestão do conhecimento aplicada tanto em nível interno, quanto às áreas externas da organização, principalmente àquelas que se relacionam ao ambiente competitivo do mercado.

De acordo com Grossman (2006) a medição sistemática do desempenho das práticas de gestão do conhecimento pode contribuir para identificar, mapear, monitorar os ativos intangíveis, padrões de fluxo de conhecimento, redes sociais, questões relativa aos conhecimentos críticos, e as melhores práticas em uma organização.

A falta de uma clara compreensão das implicações do impacto das atividades de gestão do conhecimento sobre o desempenho organizacional é amplamente visto como uma lacuna no contexto das organizações, pois uma das razões para os gestores investirem em gestão do conhecimento é a expectativa de algum retorno sobre estes investimentos (HEISIG, 2015).

Portanto, a discussão sobre o desempenho, embora longe de consenso, encontra caminhos comuns no reconhecimento da multidimensionalidade do conceito e na necessidade de separação das diferentes esferas de influência (VENKATRAMAN, RAMANUJAM, 1986; COMBS, CROOK, SHOOK, 2005). 


\section{MÉTODOS}

\subsection{CARACTERIZAÇÃO DA INSTITUIÇÃO DE ENSINO SUPERIOR EM ESTUDO}

A instituição de ensino superior trata-se da Associação Educacional Nossa Senhora Aparecida - AENSA, fundada em 1998, sendo a primeira instituição de ensino superior no município de Aparecida de Goiânia - Goiás.

Atualmente oferece um total de doze cursos: a) Cursos de graduação - bacharelados: Administração, Ciências Contábeis, Direito e Pedagogia. b) Curso de graduação tecnológica: Tecnologia em Análise e Desenvolvimento de Sistemas; Logística; Marketing; Gestão em Recursos Humanos; Gestão Comercial; Produção Publicitária; Tecnologia em Rede de Computadores e Secretariado. A instituição possui um total de 113 colaboradores sendo 90 docentes e 23 técnicos administrativos e 1.230 alunos matriculados. Os discentes não foram alvo do estudo.

\subsection{AMOSTRA}

A coleta dos dados foi realizada entre os meses de setembro a novembro de 2015 . Docentes e técnicos administrativos da instituição de ensino receberam um questionário com uma carta de apresentação explicando a natureza do estudo, bem como a permissão da direção e dos coordenadores de cursos. Os participantes foram convidados a preencher e devolver os questionários aos pesquisadores que utilizaram envelopes-resposta fechados. Foram distribuídos 113 questionários e 98 foram devolvidos, resultando em uma taxa de resposta de $87 \%$. Os respondentes representaram 16 técnicos administrativos e 82 docentes. $66 \%$ dos participantes eram do sexo masculino e o restante feminino.

\subsection{INSTRUMENTO DE PESQUISA E ANÁLISE DOS DADOS}

O instrumento de pesquisa utilizado neste estudo foi uma versão modificada da ferramenta de avaliação da gestão do conhecimento desenvolvido em 1996 pelar Arthur Andersen Consulting e o Centro de Qualidade e Produtividade Americana (APQC). Esse modelo mostra que tais práticas são apoiadas por quatro facilitadores estratégicos: liderança, cultura, tecnologia de informação e desempenho. Na visão de Coukos-Semmel (2002) esse modelo é adequado, pois oferece uma prática viável de gestão do conhecimento nas IES.

A utilização do instrumento APQC em um processo de avaliação da gestão do conhecimento permite rápida investigação, porque dispõe de um questionário sintético que 


\section{A UTILIZAÇÃO DAS PRÁTICAS DE GESTÃO DO CONHECIMENTO E FACILITADORES \\ ESTRATÉGICOS EM UMA INSTITUIÇÃO DE ENSINO SUPERIOR \\ DOI: http://dx.doi.org/10.5007/1983-4535.2018v11n2p319}

mede como a organização cria, identifica, coleciona, adapta, organiza, aplica, compartilha conhecimento (RAEUB; STHAPIT, 2001).

O questionário é dividido em duas partes. Uma com 57 itens classificada em duas dimensões: uso e importância, a outra se refere ao perfil demográfico. Os itens nas dimensões importância e utilização das práticas de gestão do conhecimento e facilitadores estratégicos consistem em afirmações descritivas utilizando a escala tipo Likert que variou de 1 = nada importante a 5 = muito importante, com a finalidade de medir o grau de importância. Para medir o grau de utilização foi adotado de 1 = não usado a 5 = extensivamente utilizado.

Precedendo a distribuição, o questionário foi testado com a participação de 10 respondentes para verificar adequação, legibilidade e abrangência do instrumento de pesquisa (PASQUALI, 2012), tendo em vista que o instrumento foi originalmente concebido para medir as práticas de gestão do conhecimento no ambiente corporativo, e adaptado para instituição de ensino superior.

Os resultados da análise de fatorial na Tabela 1 explicam, respectivamente, entre 70,$99 ; 74,99$, e 76,43 por cento; e 79,48; 78,76 e 71,83 por cento de todas as três dimensões (Geração, codificação e transferência de conhecimento) sobre o uso e importância de práticas de gestão do conhecimento. Com valores próximos de 1,00 ou superior e as cargas de 0,40 e acima foram utilizados como mínimos de corte neste estudo, tendo em vista que é uma prática comum em ciências sociais, quando o tamanho da amostra é de 100 ou mais (HAIR Jr et al. 2009).

A tabela 1 mostra também que os coeficientes para todas as práticas de gestão de conhecimento estão acima de 0,70, o que significa alta confiabilidade (SEKARAN e BOUGIE, 2010). Assim, a confiabilidade e a validade do instrumento apoiou a adequação da medida utilizada neste estudo.

Tabela 1 Resultados da análise fatorial para práticas de Gestão de Conhecimento - uso e importância

\begin{tabular}{llcccc}
\hline & & $\begin{array}{c}\text { Carga } \\
\text { Fatorial }\end{array}$ & Autovalor & $\begin{array}{c}\text { Variação } \\
\mathbf{( \% )}\end{array}$ & $\begin{array}{c}\text { Coeficiente } \\
\boldsymbol{\alpha}\end{array}$ \\
\hline $\begin{array}{l}\text { Fator 1: Geração de Conhecimento } \\
\text { Lacunas de conhecimento }\end{array}$ são & 0.827 & 2.778 & 70.99 & 0.847 \\
sistematicamente identificados. & & 0.579 & 1.136 & 79.48 & $0.847 a$ \\
$\begin{array}{l}\text { Práticas de trabalho bem definidas são } \\
\text { usadas para fechar as lacunas de }\end{array}$ & 0.844 & & & \\
conhecimento. & & 0.879 & & & \\
A instituição de ensino cria um mapa do & 0.885 & & & \\
\hline
\end{tabular}


conhecimento do seu conhecimento organizacional indicando onde a informação é localizada e como acessá-lo.

Existem diretórios de conhecimento que 0.864

listam dos empregados habilidades, 0.909 conhecimento, localização.

Os diretórios de conhecimento de outros grupos alinhados com a instituição são também divulgados.

A instituição de ensino se destaca na análise do ambiente para obter informações.

\section{Fator 2: Codificação do conhecimento}

O conhecimento tácito (os colaboradores sabem como fazer, mas não pode expressar) é avaliado na instituição de ensino. O conhecimento tácito (os colaboradores sabem como fazer, mas não pode expressar) é transferido através da instituição de ensino. A instituição de ensino possui sistemas para identificar e repassar o conhecimento interno dos colaboradores individuais.

Os conhecimentos são explicitados e acessíveis.

As bases de dados eletrônicas são acessíveis.

A maior parte dos conhecimentos dos funcionários permanece dentro da instituição de ensino ao saírem da mesma.

Fator 3: Transferência de conhecimento

A instituição de ensino formalizou a prática de transferência de boas práticas.

A instituição de ensino fornece local e ocasiões para os colaboradores falar e ouvir uns aos outros e interagir informalmente.

As bases de dados de causas e soluções de frequentes problemas encontrados são atualizadas regularmente.

As histórias de sucesso envolvendo novas abordagens são amplamente comunicadas.

As práticas de conhecimento são comunicadas rapidamente através da instituição, tornando mais fácil para transferir melhores práticas.
0.880

0.817

0.827

0.893

0.520
0.829

0.765

0.858

0.755

0.830

0.796

0.692

0.690

0.714

0.825

0.769

0.721

0.733

0.889

0.896

0.946

0.874

0.926

0.922

0.956

$\begin{array}{llll}0.513 & 1.987 & 78.76 & 0.808\end{array}$

2.857

76.43

0.814

2.744

0.879

Nota: (a) As estatísticas para a importância da dimensão são mostradas em itálico.

Da mesma forma, a Tabela 2 mostra o resumo dos resultados da análise fatorial para os facilitadores estratégicos de gestão do conhecimento sua utilização e importância usando o 


\section{A UTILIZAÇÃO DAS PRÁTICAS DE GESTÃO DO CONHECIMENTO E FACILITADORES ESTRATÉGICOS EM UMA INSTITUIÇÃO DE ENSINO SUPERIOR \\ DOI: http://dx.doi.org/10.5007/1983-4535.2018v11n2p319}

método de rotação varimax, sendo que nenhum dos itens fora eliminado, pois eles mantêm uma carga de 0,40 e acima. Os resultados da análise fatorial explicam entre 79,87 e 73,22 por cento; e 75,99 e 69,09 por cento, respectivamente, de todas as quatro dimensões de facilitadores estratégicos, tanto para uso quanto importância.

Tabela 2 Resultados da análise fatorial para os facilitadores estratégicos - uso e importancia

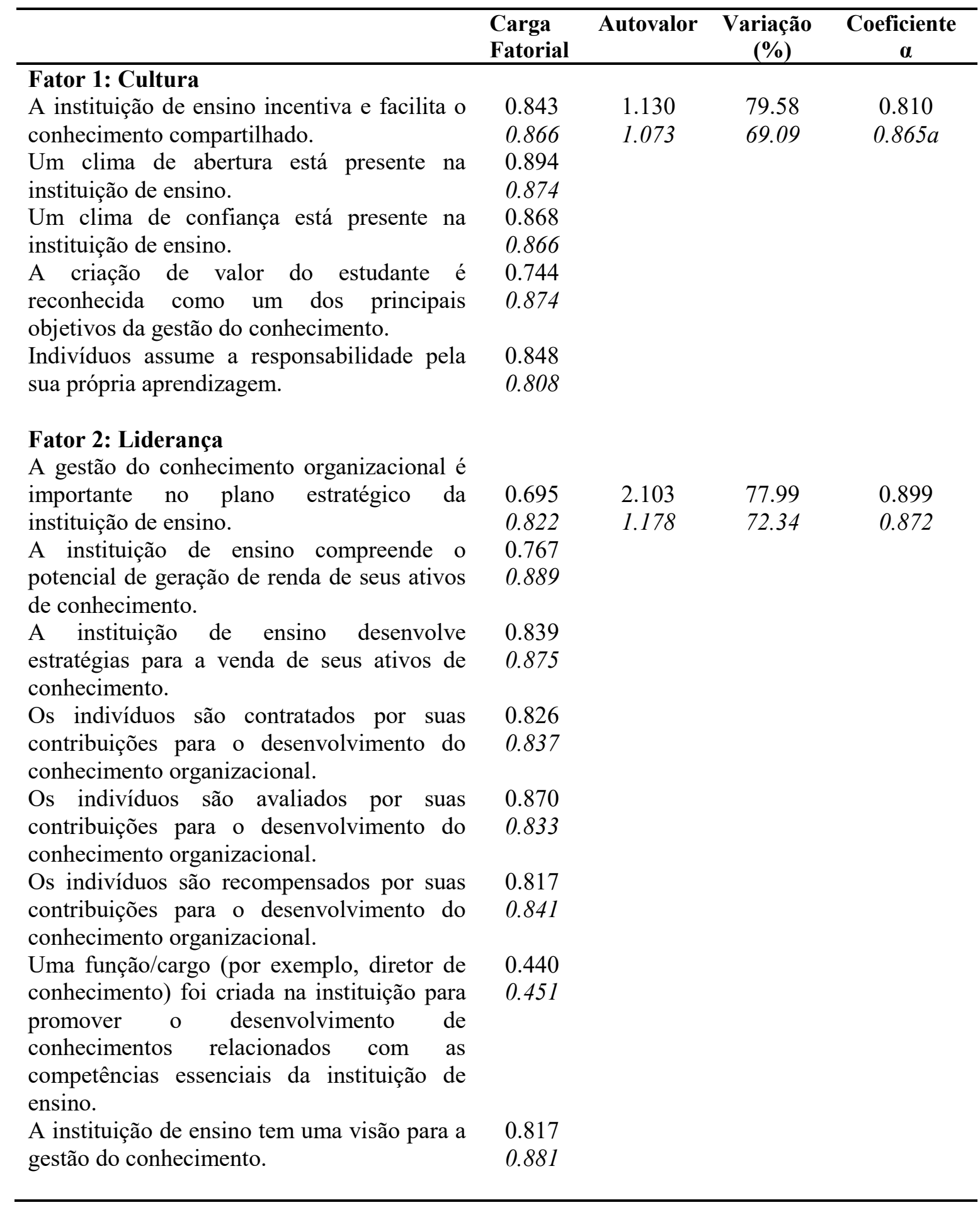




\section{A UTILIZAÇÃO DAS PRÁTICAS DE GESTÃO DO CONHECIMENTO E FACILITADORES \\ ESTRATÉGICOS EM UMA INSTITUIÇÃO DE ENSINO SUPERIOR \\ DOI: http://dx.doi.org/10.5007/1983-4535.2018v11n2p319}

\begin{tabular}{|c|c|c|c|c|}
\hline & $\begin{array}{l}\text { Carga } \\
\text { Fatorial } \\
\end{array}$ & Autovalor & $\begin{array}{c}\text { Variação } \\
(\%)\end{array}$ & $\begin{array}{c}\text { Coeficiente } \\
\alpha\end{array}$ \\
\hline \multicolumn{5}{|l|}{ Fator 3: Tecnologia da informação } \\
\hline A tecnologia da informação (TI) liga um a & 0.644 & 2.277 & 79.87 & 0.788 \\
\hline $\begin{array}{l}\text { outro todos os membros da instituição de } \\
\text { ensino. }\end{array}$ & 0.889 & 1.997 & 75.99 & 0.810 \\
\hline A tecnologia da informação (TI) vincula & 0.574 & & & \\
\hline $\begin{array}{l}\text { todos os membros da instituição com } \\
\text { relevante público. }\end{array}$ & 0.844 & & & \\
\hline A (TI) cria um banco de dados que é & 0.785 & & & \\
\hline acessível a toda a instituição de ensino. & 0.915 & & & \\
\hline A (TI) coloca a instituição de ensino mais & 0.813 & & & \\
\hline próxima dos seus alunos. & 0.903 & & & \\
\hline A (TI) é projetado para ajudar os & 0.868 & & & \\
\hline colaboradores a trabalhar de forma eficiente. & 0.985 & & & \\
\hline A (TI) é projetado para ajudar os & 0.892 & & & \\
\hline colaboradores a tomar melhores decisões. & 0.865 & & & \\
\hline A instituição de ensino atualiza e substitui de & 0.836 & & & \\
\hline forma contínua hardware e software. & 0.870 & & & \\
\hline As informações atingem tomadores de & 0.834 & & & \\
\hline decisão apropriados em tempo hábil. & 0.877 & & & \\
\hline \multicolumn{5}{|l|}{ Fator 4. Desempenho } \\
\hline A instituição de ensino vincula a gestão do & 0.591 & 1.211 & 73.22 & 0.821 \\
\hline conhecimento com o orçamento alocado. & 0.901 & 1.453 & 75.23 & 0.855 \\
\hline O relatório anual da instituição inclui uma & 0.896 & & & \\
\hline $\begin{array}{l}\text { avaliação de como o capital do } \\
\text { conhecimento tem contribuído para uso das } \\
\text { atividades fins. }\end{array}$ & 0.828 & & & \\
\hline A instituição tem desenvolvido um conjunto & 0.900 & & & \\
\hline $\begin{array}{l}\text { específico de indicadores de gestão do } \\
\text { conhecimento. }\end{array}$ & 0.915 & & & \\
\hline O sistema de medição mede os ativos & 0.932 & & & \\
\hline intangíveis (por exemplo: capital intelectual) & 0.930 & & & \\
\hline A instituição aloca recursos para os esforços & 0.850 & & & \\
\hline $\begin{array}{l}\text { que comprovadamente aumentam a sua base } \\
\text { de conhecimento. }\end{array}$ & 0.889 & & & \\
\hline
\end{tabular}

Nota: (a) As estatísticas para a importância da dimensão são mostradas em itálico.

\section{RESULTADOS}

A tabela 3 mostra a pontuação e o desvio padrão médios para o grau de importância e uso de práticas de gestão de conhecimento. As pontuações médias para graus de importância estão acima de 4.083, indicando que as práticas são consideradas importantes.

A codificação do conhecimento teve a maior média, seguido de transferência, e posteriormente, geração de conhecimento. No entanto, para o grau de utilização, as notas variaram entre 3.521 e 3.528 , o que implica a utilização média das práticas de gestão de 


\section{A UTILIZAÇÃO DAS PRÁTICAS DE GESTÃO DO CONHECIMENTO E FACILITADORES \\ ESTRATÉGICOS EM UMA INSTITUIÇÃO DE ENSINO SUPERIOR \\ DOI: http://dx.doi.org/10.5007/1983-4535.2018v11n2p319}

conhecimento. A transferência de conhecimento teve a maior média, seguidos de geração e codificação do conhecimento.

Tabela 3 Grau de importância e uso de práticas de gestão de conhecimento

\begin{tabular}{ccccccccc}
\hline $\begin{array}{c}\text { Práticas de } \\
\text { Gestão de }\end{array}$ & \multicolumn{2}{c}{$\begin{array}{c}\text { Grau de } \\
\text { Importância }\end{array}$} & \multicolumn{2}{c}{ Grau de Utilização } & & & \\
\cline { 2 - 8 } Conhecimento & Média & SD & Ranking & Média & SD & Ranking & $\begin{array}{c}t \text { - } \\
\text { value }\end{array}$ & $\begin{array}{c}\text { Significância } \\
\text { (Bicaudal) }\end{array}$ \\
Geração & 4.083 & 0.7053 & 3 & 3.524 & 0.8464 & 2 & 5.723 & 0.000 \\
Codificação & 4.853 & 0.6754 & 1 & 3.521 & 0.8201 & 3 & 6.468 & 0.000 \\
Transferência & 4.136 & 0.6700 & 2 & 3.528 & 0.8452 & 1 & 6.190 & 0.000 \\
\hline
\end{tabular}

A tabela 4 mostra a pontuação e desvio padrão médio para o grau de importância e utilização dos principais facilitadores estratégicos da gestão do conhecimento pesquisados. As pontuações significativas para grau de importância estão acima de 3,96, indicando que os facilitadores são considerados importantes.

A tecnologia da informação (TI) teve a maior média, seguida de liderança, desempenho e cultura. Quanto ao grau de utilização, também a tecnologia de informação teve a maior média, seguida de liderança, desempenho e cultura.

Tabela 4 Grau de importância e uso de facilitadores estratégicos de gestão de conhecimento

\begin{tabular}{|c|c|c|c|c|c|c|c|c|}
\hline \multirow{2}{*}{$\begin{array}{l}\text { Facilitadores de } \\
\text { Gestão de } \\
\text { Conhecimento }\end{array}$} & \multicolumn{2}{|c|}{$\begin{array}{c}\text { Grau de } \\
\text { Importância } \\
\end{array}$} & \multicolumn{3}{|c|}{ Grau de Utilização } & \multirow[b]{2}{*}{ Ranking } & \multirow[b]{2}{*}{$\begin{array}{c}t- \\
\text { value }\end{array}$} & \multirow[b]{2}{*}{$\begin{array}{l}\text { Significância } \\
\text { (Bicaudal) }\end{array}$} \\
\hline & Média & SD & Ranking & Média & SD & & & \\
\hline Cultura & 3.96 & 0.829 & 4 & 3.71 & 0.667 & 4 & 3.261 & 0.001 \\
\hline Liderança & 4.14 & 0.646 & 2 & 3.93 & 0.646 & 2 & 3.214 & 0.002 \\
\hline TI & 4.39 & 0.648 & 1 & 4.01 & 0.667 & 1 & 5.673 & 0.000 \\
\hline Desempenho & 4.14 & 0.751 & 3 & 3.84 & 0.868 & 3 & 3.533 & 0.001 \\
\hline
\end{tabular}

Os escores médios de importância e uso de práticas de gestão de conhecimento foram agregados separadamente e a respectiva correlação com cada um dos facilitadores estratégicos foi examinada por meio do teste do coeficiente de correlação de Pearson, com nível de significância de 5\%. Segundo Triola (2008) o coeficiente linear de Pearson mede a intensidade da relação linear entre os valores quantitativos emparelhados em uma amostra. A tabela 5 demonstra que a utilização de práticas de gestão do conhecimento não está significativamente associada à utilização de qualquer um dos facilitadores estratégico, embora as correlações sejam positivas. No entanto, coeficientes significativos de correlação são 


\section{A UTILIZAÇÃO DAS PRÁTICAS DE GESTÃO DO CONHECIMENTO E FACILITADORES \\ ESTRATÉGICOS EM UMA INSTITUIÇÃO DE ENSINO SUPERIOR \\ DOI: http://dx.doi.org/10.5007/1983-4535.2018v11n2p319}

registrados entre a importância do desempenho, liderança e de tecnologia da informação (TI) com a importância das práticas de gestão do conhecimento.

Tabela 5 Correlação entre o uso e a importância de práticas de gestão de conhecimento e uso e importância dos principais facilitadores estratégicos.

\begin{tabular}{|c|c|c|c|c|}
\hline $\begin{array}{l}\text { Facilitadores } \\
\text { Estratégicos }\end{array}$ & Cultura & Liderança & $\begin{array}{ll}\text { Tecnologia } & \text { da } \\
\text { Informação } & \\
\end{array}$ & Desempenho \\
\hline \multicolumn{5}{|c|}{$\begin{array}{l}\text { Práticas de Gestão } \\
\text { de Conhecimento }\end{array}$} \\
\hline Uso & 0.059 & 0.048 & 0.098 & 0.066 \\
\hline Importância & 0.042 & $273 * *$ & $185^{*}$ & $289 * *$ \\
\hline
\end{tabular}

Nota: *, **A correlação é significativa ao nível de 0,05 e 0,01 (bicaudal), respectivamente.

\section{DISCUSSÕES}

Ao analisar o grau de importância e utilização das práticas de gestão do conhecimento e facilitadores estratégicos na IES pesquisada, os respondentes afirmam que todas as práticas de gestão do conhecimento são importantes para a IES, bem como destacaram a importância dos quatro facilitadores estratégicos embora a média para cultura seja ligeiramente inferior a 4,00 .

Assim, pode-se inferir que os resultados da pesquisa estão coadunados com estudos anteriores, onde as práticas de gestão do conhecimento e facilitadores estratégicos são fatores críticos para sucesso de sua implementação (COUKOS-SEMMEL, 2002; CHONG, 2006; CHONG e LIN, 2009; FERRARESI, QUANDT, FREGA, SANTOS, 2012; 2014). Também se pode concluir que os setores de ensino e empresarial partilham da mesma preocupação sobre a importância das práticas de gestão do conhecimento e as estratégias facilitadoras dessas práticas.

O estudo atual revela que a utilização da tecnologia de informação teve a maior média, seguido de liderança, desempenho e cultura. Alguns estudos, no entanto, alegaram que a cultura organizacional é mais importante do que o suporte de tecnologia de informação para a implementação de práticas de gestão do conhecimento. Por exemplo, Gold, Malhorta, Segars (2001) descobriram que a cultura tem ponderação mais elevada do que a tecnologia em termos de capacidades de infraestrutura de conhecimento que serve como facilitador de gestão do conhecimento visando otimizar a eficácia organizacional. Entretanto, estudos de Lee e Lee 


\section{A UTILIZAÇÃO DAS PRÁTICAS DE GESTÃO DO CONHECIMENTO E FACILITADORES \\ ESTRATÉGICOS EM UMA INSTITUIÇÃO DE ENSINO SUPERIOR \\ DOI: http://dx.doi.org/10.5007/1983-4535.2018v11n2p319}

(2007) descobriram que o suporte de tecnologia de informação teve um efeito mais forte nos processos de gestão do conhecimento do que na cultura. Esses diferentes achados refletem o fato de que a tecnologia da informação pode estar desempenhando um papel cada vez mais importante no contexto da gestão do conhecimento.

Em relação ao segundo objetivo do estudo, verificar a existência de dicotomias entre o grau de importância e uso das práticas de gestão do conhecimento e facilitadores estratégicos. Os resultados indicam que muito precisa ser feito na IES pesquisada para garantir maior intensidade de práticas de gestão do conhecimento. Da mesma forma, a importância e a utilização dos principais facilitadores estratégicos retratam diferenças significativas, sugerindo que a instituição necessita usar os facilitadores para apoiar suas práticas organizacionais.

A alta administração tem papel importante no gerenciamento do conhecimento para que as práticas avancem. Uma sugestão é implantar o departamento ou setor de gestão do conhecimento na sua estrutura organizacional, a fim de elevar sua importância. A estrutura organizacional pode encorajar ou inibir a disseminação do conhecimento; esta estrutura deve incluir a centralização e a formalização das iniciativas da gestão do conhecimento (CHONG E LIN, 2009). O grau e a natureza da partilha de conhecimentos dependem das iniciativas individuais de professores ou de pequenos grupos de pesquisadores que compartilham com interesses semelhantes (CUFFA, ROJO e MELLO, 2014).

No que diz respeito ao terceiro objetivo, identificar as relações entre as práticas de gestão do conhecimento e os facilitadores estratégicos em termos de importância e uso em instituições de ensino superior. Os resultados da correlação de Pearson sugerem que não existe uma associação significativa entre o uso de facilitadores estratégicos e de práticas de gestão do conhecimento na IES pesquisada. Como a instituição ainda é relativamente nova no campo do ensino superior, a adoção de facilitadores de gestão de conhecimento pode não ter impactos substancial sobre a execução real dos processos. Também pode ser que os facilitadores sejam utilizados para apoiar e sustentar a implementação de outras iniciativas na IES, reduzindo assim o seu efeito sobre a prática de gestão do conhecimento.

Por outro lado, o teste de correlação confirma que desempenho, liderança e tecnologia da informação são importantes para as práticas de gestão do conhecimento. Ou seja, os processos de conhecimento (geração, codificação e transferência) exigem a presença de tecnologia da informação como suporte de gestão e medição de desempenho com base em 


\section{A UTILIZAÇÃO DAS PRÁTICAS DE GESTÃO DO CONHECIMENTO E FACILITADORES \\ ESTRATÉGICOS EM UMA INSTITUIÇÃO DE ENSINO SUPERIOR \\ DOI: http://dx.doi.org/10.5007/1983-4535.2018v11n2p319}

atividades de conhecimento para que eles sejam bem sucedidos, conforme preconizados por Derntl e Motschning-Pitrik (2005) em seus estudos. Um gerenciamento forte e o apoio dos lideres é essencial para utilização das práticas de gestão do conhecimento nas rotinas de trabalho das instituições de ensino superior. A liderança é uma condição essencial para que os esforços de gestão do conhecimento possam ser explorados (VON KROGH et al., 2011).

Outra questão é a relação de importância entre a cultura organizacional e as práticas de gestão do conhecimento que tem sido de alguma forma subavaliado pela IES pesquisada. A cultura organizacional carrega a base genética que irá moldar a evolução de comportamentos, atitudes, normas e crenças das pessoas em uma organização (NELSON e QUICK, 2011). Assim, uma cultura ideal para o conhecimento deve ser criada e alimentada continuamente de modo que as práticas de gestão do conhecimento sejam uma rotina na instituição de ensino.

Existem várias limitações para este estudo. Algumas limitações têm relação com a amostra, outras com o instrumento de coleta de dados e com o alcance dos resultados. Uma forte limitação foram os dados coletados em uma única instituição de ensino privado, portanto os resultados podem ser consideravelmente afetados pelas características de regionalidade da amostra. Isto coloca desafios para a generalização de nossos resultados.

\section{CONCLUSÕES}

Esta pesquisa demonstrou como são utilizados os facilitadores estratégicos que apoiam as práticas de gestão do conhecimento em instituição de ensino superior, bem como o grau de importância.

Os resultados demonstraram que existem diferenças significativas entre a importância e o uso das práticas de gestão de conhecimento. A prática de codificação do conhecimento teve maior importância, seguido de transferência e da geração de conhecimento. Entretanto, quanto ao grau de utilização, a transferência de conhecimento teve a maior média, seguido de geração e codificação do conhecimento.

Este estudo também revelou que a tecnologia da informação, a liderança, o desempenho e a cultura organizacional são facilitadores estratégico que promovem a otimização das praticas de gestão de conhecimento nas organizações, em específico, de ensino superior. Espera-se que os resultados apresentados contribuam para que os gestores possam realizar ações corretivas a fim avançar na consolidação de práticas de gestão de conhecimento em instituição de ensino. Uma boa gestão do conhecimento pode proporcionar oportunidades 
de aprendizagem organizacional criando maior vantagem competitiva para as organizações (MELTON et al. (2006).

Como pesquisas futuras sugerem-se os seguintes estudos: a) Investigar outras instituições de ensino públicas e privadas para validar os argumentos apresentados nesta pesquisa e que considere outros facilitadores: treinamento de colaboradores, envolvimento dos trabalhadores, trabalhos em equipe, benchmarking e estrutura de conhecimento organizacional; b) Verificar como os fatores humanos, culturais, técnicos e específicos das organizações afetam o desenvolvimento da gestão do conhecimento; c) Verificar quais são as principais ferramentas e práticas utilizadas pelas organizações empresariais para melhorar as diferentes fases do processo de desenvolvimento da gestão do conhecimento.

\section{REFERÊNCIAS}

ALBERTIN, A. L. Valor estratégico dos projetos de tecnologia de informação. Revista de Administração de Empresas, 41(3), 42-50, 2001.

ARTHUR ANDERSEN. The American Productivity and Quality Centre - APQC. The KM assessment tool: external benchmarking version. Arthur Anderson, St Charles, IL, Winter, 1996.

ANTONY, J. P; BHATTACHARYYA, S. Measuring organizational performance and organizational excellence of SMEs - Part 2: an empirical study on SMEs in India. Measuring Business Excellence. v.14, n. 3, p. 42-52, 2010.

BARBUTO, J.E. Motivation and transactional, charismatic, and transformational leadership: A test of antecedents. Journal of Leadership and Organizational Studies, 11, 4, 2005.

BRENNAND, E.G de G. Hipermíidia e novas engenharias cognitivas nos espaços de formação. In: SILVA, A. M.M. et. al. Políticas Educacionais, Tecnologias e Formação do Educador: repercussões sobre a Didática e as Práticas de Ensino. Recife: ENDIPE, 2006.

BUCHELE, G T. et al. Contribuição das Comunidades de Prática para o Processo de Autoavaliação em uma Instituição de Educação Superior do Segmento Privado. Revista Gestão Universitária na América Latina, v. 9, n. 1, p. 97-119, 2016.

CARVALHO, R.B. Tecnologia da informação aplicada à gestão do conhecimento. Belo Horizonte: C/Arte, 2003.

COMBS, J. G., CROOK, T. R., SHOOK, C. L. The dimension of organizational performance and its implications for strategic management research. In: Research Methodology in Strategy and Management, San Diego, CA: Elsevier. p. 259-286, 2005. 
CHONG, C.W; HOLDEN, T; WILHELMIJ, P; SCHMIDT, R. A. Where does knowledge management add value? Journal of Intellectual Capital, vol. 1, n. 4, pp. 366-80, 2000. $\mathrm{CHONG}$, S. C. KM critical success factors: a comparison of perceived importance versus implementation in Malaysian ICT companies. The Learning Organisation, vol. 13, n. 3, pp. 230-56, 2006.

CHONG, S.C; LIN, B. Implementation level of knowledge management critical success factors in Malaysia. International Journal of Innovation and Learning, vol. 6 n. 5, pp. 493-516, 2009.

CHOO, C. W. (2003). A Organização do Conhecimento. São Paulo: Editora Senac, 2003.

COUKOS-SEMMEL, E.D. Knowledge management: processes and strategies used in United States research universities. unpublished academic dissertation, Florida Atlantic University, Boca Raton, FL, 2002.

CUFFA, D; ROJO, C. A; MELLO, G. R. Gestão do conhecimento no ensino superior: um estudo com acadêmicos do curso de Administração. Revista Carta Capital - Eletrônica (RCCe), v. 12, n. 2, abr./jun, 2014.

DAVENPORT, T.; PRUSAK, L. Conhecimento Empresarial: Como as Organizações Gerenciam Seu Capital. 12. ed., Rio de Janeiro: Elsevier, 2003.

DERNTL, M.; MOTSCHNIG-PITRIK, R. THE rule of structure, patterns, and people in blended learning. The Internet and Higher Education, v. 8, n. 2, p. 111- 130, 2005.

EIRIZ, V.; SIMÕES, J. GONÇALVES, M. Obstáculos à gestão do conhecimento nas escolas de gestão e economia do ensino superior público em Portugal. Comportamento Organizacional e Gestão, v. 13, n. 2, p. 153-167, 2007.

FERRARESI, A. A; QUANDT, C. O; FREGA, J. R; SANTOS, S. A. Knowledge management and strategic orientation: leveraging innovativeness and performance. Journal of Knowledge Management, v. 16, p. 688-701, 2012.

FERRARESI, A. A; QUANDT, C. O; FREGA, J. R;, SANTOS, S. A. Os impactos da gestão do conhecimento na orientação estratégica, na inovatividade e nos resultados organizacionais: uma survey com empresas instaladas no Brasil. RAM. Revista de Administração Mackenzie, v. 15, p. 199-231, mar./abril, 2014.

GOLD, A.H; MALHORTA, A; SEGARS, A.H. Knowledge management: an organisation capabilities perspective. Journal of Management Systems, vol. 18, n. 1, p. 185-214, 2001.

GROSSMAN, M. An overview of KM as assessment. Journal of American Academy of Business, Cambridge, vol. 8, n. 2, p. 23-38, 2006.

HARTUNG, K; OLIVEIRA, M. Communities of practice: creating and sharing knowledge. Revista de Gestão, 20 (3), 407-422, 2013. 
HAIR JR. F; BLACK, W. C; BABIN, B. J; ANDERSON, R. E; TATHAM, R. L. (2009). Análise multivariada de dados. (A. S. Sant'Anna, Trad.). Porto Alegre: Bookman, 2009. HEISIG, $\mathrm{P}$. Future research in knowledge management: results from the global knowledge research network study, in Bolisani, E. and Handzic, M. (Eds), Advances in Knowledge Management, Springer International Publishing, available at: http://link.springer.com/chapter/10.1007/978-3-319-09 501-1_7, pp. 151-182, 2015.

HEYDARI, M; AHSANI,G; DANAI, H. Model key elements of knowledge management to enhance creativity and organizational learning (the case of the Standards and Industrial Research of Iran), Journal of Teknologi Tanaman, 12, 367 - 380, 2015

HOLSAPPLE, C.W; JOSHI, K.D. Knowledge management: a threefold framework. Information Society, vol. 18, n. 1, pp. 47-64, 2002.

HUSEEIN, N; MOHAMAD, A; NOORDIN, F; ISHAK, N, A. Learning Organization and its Effect on Organizational Performance and Organizational Innovativeness: A Proposed Framework for Malaysian Public Institutions of Higher Education. Procedia - Social and Behavioral Sciences, v. 130. p. 299 - 304, 2014.

KENSKI, V. M. Educação e tecnologias: o novo ritmo da informação. Campinas, SP: Papirus, 2007.

LEE, Y.C; LEE, S.K. Capabilities, processes and performance of knowledge management: a structural approach. Human Factors and Ergonomics in Manufacturing, vol. 17, n. 1, pp. 21-41, 2007.

LOPES, M. C; FIALHO, F. A. P. Liderança no contexto da sociedade do conhecimento: uma revisão sistemática. Revista da UNIFEBE, 1(13), 1-19, 2014.

MARIN, A; CORDIER, J; HAMEED, T. Reconciling ambiguity with interaction: implementing formal knowledge strategies in a knowledge-intensive organization. Journal of Knowledge Management, vol. 20 Iss 5 pp. 959 - 979, 2016.

MAIER, R.; REMUS, U. Defining process-oriented knowledge management strategies. Knowledge and Process Management, v. 9, n. 2, p. 103-118, 2002.

MELTON, C. E; CHEN, J. C. H; LIN, B.. Organisational knowledge and learning: leveraging it to accelerate the creation of competitive advantages. International Journal of Innovation and Learning, vol. 3, n. 3, pp. 254-66, 2006.

MINTZBERG, H. Covert leadership: notes on managing professionals. Harvard Business Review. 36 (6), 140-147, 1998.

NEELY, A. The evolution of performance measurement research: developments in the last decade and a research agenda for the next. International Journal of Operations \& Production Management, v. 25, n.12, p. 1264 -1277, 2005. 
NELSON, D. L; QUICK, J. C. Understanding Organizational Behavior. Belmont, CA: Cengage South-Western, 2011.

PASQUALI, L. Análise Fatorial para pesquisadores. 1ed. Brasília: LabPAMEditora, 2012.

PIRES, J. C. S; MACÊDO, K. B. Cultura organizacional em organizações públicas no Brasil. Revista de Administração Pública. Rio de Janeiro, v.40, p. 81-105, 2006.

RAEUB, S. P.; STHAPIT, B. Towards a taxonomy of approaches for measuring organizational knowledge. Research and Practice in Human Resource Management. 9(1), 2001, 139-155, 2001.

SCHATZKI, T. R. Peripheral Vision: The sites of organizations. Organization Studies. v. 26, n. 3, p. 465-84, 2005.

SHARIMLLAH DEVI, R; CHONG, S.C; LIN, B.. Organisational culture and KM practices from the perspective of institutions of higher learning. International Journal of Management in Education, vol. 1, ns 1/2, pp. 57-79, 2007.

SEKARAN, U; BOUGIE, R. Research Methods for Business: A Skill Building Approach, John Wiley \& Sons Inc, New York, NY, 2010.

TAYLOR, C. R. (2004). Retention leadership. T and D, v. 58 (3), p. 40-45, 2004.

TIPPINS, M.J. Implementing knowledge management in academia: teaching the teachers. The International Journal of Educational Management, vol. 17, n. 7, pp. 339-45, 2003. TRICE, H. M; BEYER, J. M. Studying organizational cultures through rites and ceremonials. Academy of Management Review, V. 9 (4), p. 653-669, 1986.

TRIOLA, F, M. Introdução à Estatística, 10 ed. Rio de janeiro, RJ: LTC, 2008.

VENKATRAMAN, N., RAMANUJAM, V. Measurement of Business Performance in Strategy Research: A Comparison of Approaches. Academy of Management Review. v. 11, n. 4 , p. $801-814,1986$.

VON KROGH, G;, NONAKA, I; RECHSTEINER, L. Leadership in organizational knowledge creation. A review and framework', Journal of Knowledge Management Studies, vol. 49, pp. 240-277, 2011. 